\title{
Advanced imaging in acute ischemic stroke
}

\author{
Craig Kilburg, MD, J. Scott McNally, MD, PhD, ${ }^{2}$ Adam de Havenon, MD, ${ }^{3}$ Philipp Taussky, MD, ${ }^{1}$ \\ M. Yashar S. Kalani, MD, PhD, ${ }^{1}$ and Min S. Park, MD1
}

Departments of ${ }^{1}$ Neurosurgery, ${ }^{2}$ Neuroradiology, and ${ }^{3}$ Neurology, University of Utah, Salt Lake City, Utah

\begin{abstract}
The evaluation and management of acute ischemic stroke has primarily relied on the use of conventional CT and MRI techniques as well as lumen imaging sequences such as CT angiography (CTA) and MR angiography (MRA). Several newer or less-established imaging modalities, including vessel wall MRI, transcranial Doppler ultrasonography, and 4D CTA and MRA, are being developed to complement conventional CT and MRI techniques. Vessel wall MRI provides high-resolution analysis of both extracranial and intracranial vasculature to help identify previously occult lesions or characteristics of lesions that may portend a worse natural history. Transcranial Doppler ultrasonography can be used in the acute setting as a minimally invasive way of identifying large vessel occlusions or monitoring the response to stroke treatment. It can also be used to assist in the workup for cryptogenic stroke or to diagnose a patent foramen ovale. Fourdimensional CTA and MRA provide a less invasive alternative to digital subtraction angiography to determine the extent of the clot burden and the degree of collateral blood flow in large vessel occlusions. Along with technological advances, these new imaging modalities are improving the diagnosis, workup, and management of acute ischemic stroke- roles that will continue to expand in the future.
\end{abstract}

https://thejns.org/doi/abs/10.3171/2017.1.FOCUS16503

KEY WORDS ischemic stroke; vessel wall MRI; transcranial Doppler ultrasound; four-dimensional CT angiography; four-dimensional MR angiography; imaging

$\mathrm{V}$ ARIOUS imaging modalities are routinely used in the diagnosis and management of acute ischemic stroke, including CT and MRI. In addition to confirming the presence of ischemic stroke, these techniques are useful for characterizing tissue perfusion. They allow separation of the core infarct from reversible ischemic tissue at risk, thereby indicating the potential for intervention or clot extraction. In addition, lumen imaging-CT angiography (CTA), MR angiography (MRA), or digital subtraction angiography (DSA) - is often performed in the evaluation of stroke sources. These imaging modalities not only identify the location of large vessel occlusions for the intervention team, but also establish the underlying stroke etiology. ${ }^{1}$ Although these techniques offer many benefits for ischemic stroke characterization, advanced imaging techniques that can better identify stroke prognosis and underlying cause have been developed more recently.

Newly developed and refined techniques, such as vessel wall (VW)-MRI, transcranial Doppler (TCD) ultrasonography, and 4D CTA and MRA, may further aid the workup and management of acute ischemic stroke. In the extracranial vasculature, VW-MRI can identify stroke sources invisible by lumen imaging by detecting features of these lesions, such as carotid intraplaque hemorrhage (IPH). Carotid plaques with MRI-detected IPH (American Heart Association Type VIb plaque) present a risk of stroke not only from the degree of stenosis, but also from the IPH. ${ }^{15,17,39}$ Furthermore, VW-MRI is now used intracranially and identifies culprit lesions more easily than lumen imaging does, as in the case of symptomatic nonstenotic intracranial atherosclerotic disease or vasculitis. ${ }^{11}$ Transcranial Doppler ultrasonography is a minimally invasive modality that can be used to identify asymptomatic emboli and isolate the vessel of origin for emboli. It can also aid in evaluating cryptogenic stroke by detecting a patent foramen ovale (PFO) or in identifying emboli in the setting of symptomatic spontaneous dissections. ${ }^{5,8,20}$ Whereas these modalities are used primarily to determine the cause of an acute ischemic stroke, 4D CTA and MRA can be more helpful in identifying the true infarct core and potential

ABBREVIATIONS CTA = CT angiography; DSA = digital subtraction angiography; ICA = internal carotid artery; IPH = intraplaque hemorrhage; $L R N C=$ lipid-rich necrotic core; $M C A$ = middle cerebral artery; $M E S=$ microembolic signal; MPRAGE = magnetization-prepared rapid acquisition gradient echo; MRA = MR angiography; PFO = patent foramen ovale; PICA = posterior inferior cerebellar artery; TCD = transcranial Doppler; TOF = time of flight; TTE = transthoracic echocardiography; VW = vessel wall. SUBMITTED November 29, 2016. ACCEPTED January 5, 2017.

INCLUDE WHEN CITING DOI: 10.3171/2017.1.FOCUS16503. 

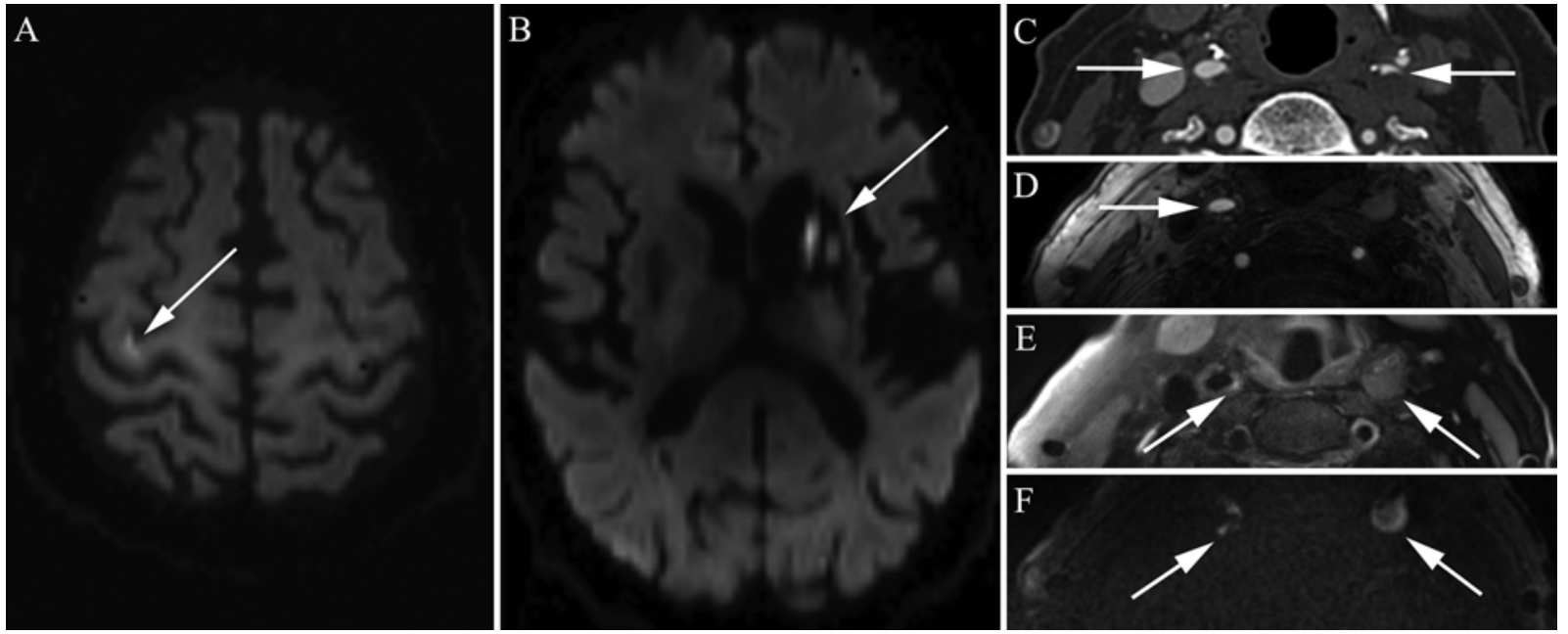

FIG. 1. An 84-year-old man with symptomatic recurrent stroke related to bilateral unstable carotid plaque had hypertension, diabetes, and hyperlipidemia, for which he was on maximal medical therapy including dual antiplatelets (aspirin and clopidogrel), antihypertensive medication (carvedilol), and a lipid-lowering agent (simvastatin). The patient's initial stroke was in the right MCA territory in the right frontal lobe (A, arrow). Three months later, the patient had another stroke in the left MCA territory (B, arrow). Computed tomography angiography showed bilateral carotid bifurcation plaques with $46 \%$ stenosis on the right and $70 \%$ stenosis on the left according to the North American Symptomatic Carotid Endarterectomy Trial criteria, with mixed soft and calcified plaque (C, arrows). Magnetic resonance imaging demonstrated flow-related enhancement that was more easily seen on the right than the left on 3D TOF images (D, arrow), a large left and small right LRNC on T2-weighted images (E, arrows), and left greater than right carotid IPH on MPRAGE images ( $F$, arrows).

collateral circulation to an area of ischemia. This information can help to guide stroke intervention and more accurately predict the outcome after stroke. ${ }^{24}$ We present a review of these advanced imaging modalities in the diagnosis and management of acute ischemic stroke.

\section{Vessel Wall MRI}

A rapidly evolving imaging technique, VW-MRI has many different uses in the workup for acute ischemic stroke. This imaging protocol includes MRA and multicontrast MRI sequences that suppress the signal from adjacent tissue and blood to highlight VW pathologies of both intracranial and extracranial vessels (Figs. 1-5). ${ }^{9}$ This can provide a wealth of new information that timeof-flight (TOF) MRI sequences and other luminal imaging techniques cannot deliver.
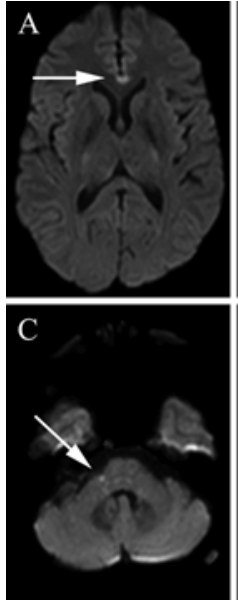
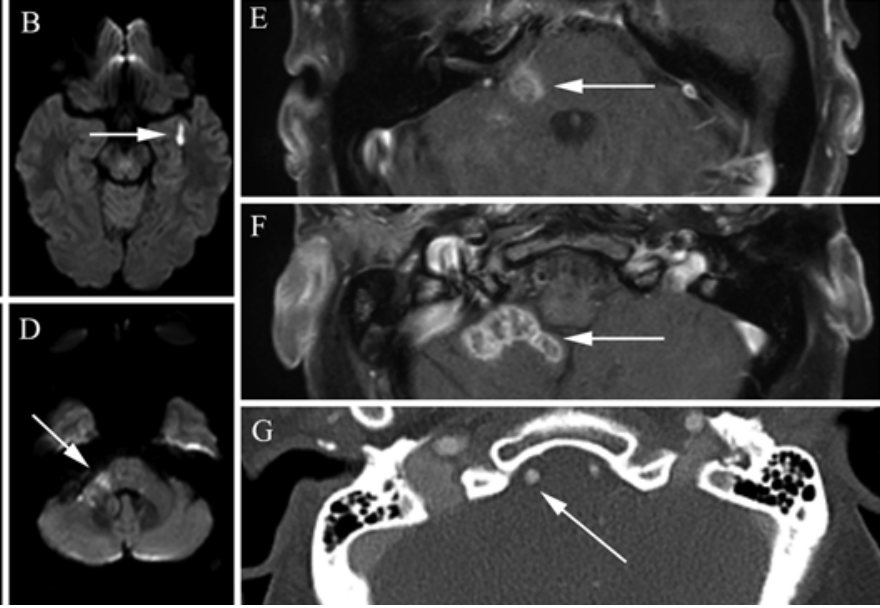

FIG. 2. A 41-year-old woman with morbid obesity, hypertension, and untreated diabetes with a hemoglobin A1c of $8.5 \%$ presented with multiple enhancing brain lesions that also showed diffusion restriction (A-C, arrows) and were initially thought to represent demyelination. The lesion in the right brainstem and cerebellum increased in size over 2 weeks (D, arrow). The largest right cerebellum and brainstem lesion showed enhancement with contrast administration ( $E$ and $F$, arrows). Computed tomography angiography was initially thought to be negative for any vascular pathology, but no right posterior inferior cerebellar artery (PICA) was detected (G). A cerebellar lesion specimen was subsequently obtained for biopsy, and pathological analysis revealed an infarct. Vessel wall T2-weighted MR images demonstrated an occluded right PICA ( $H$, horizontal arrow) and an eccentric T2-hyperintense plaque (slanted arrow) in the $\mathrm{V}_{4}$ segment proximal to the PICA occlusion. Precontrast (I) and postcontrast (J) DANTE T1 SPACE images demonstrate $\mathrm{V}_{4}$ segment $\mathrm{VW}$ enhancement (arrow) indicating actively inflamed plaque. 

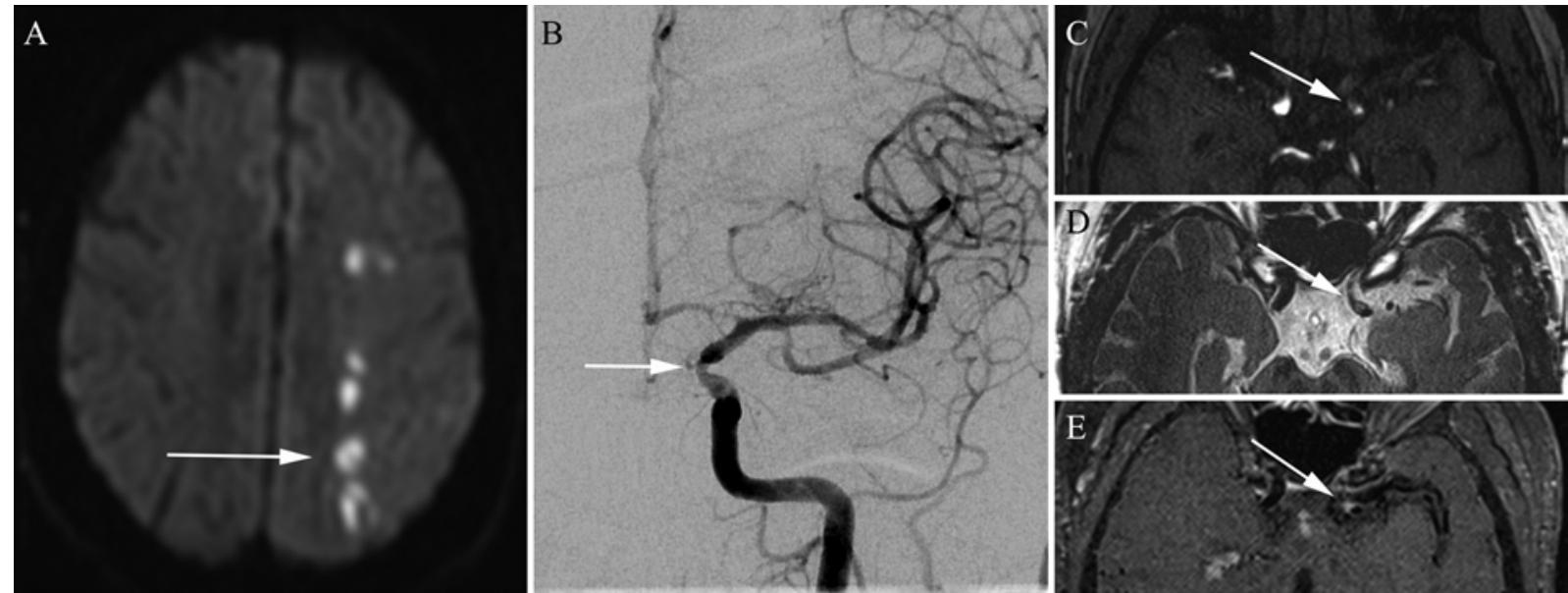

FIG. 3. An 80-year-old woman with a symptomatic recurrent left MCA distribution stroke and actively inflamed left intracranial carotid plaque had hypertension, diabetes, and hyperlipidemia, for which she was on maximal medical therapy including dual antiplatelets (aspirin and clopidogrel), antihypertensive medication (lisinopril), and a lipid-lowering agent (atorvastatin). The patient had recent and recurrent infarcts in the left ICA distribution (A, arrow). Digital subtraction angiography revealed approximately $50 \%$ narrowing of the left intracranial ICA ophthalmic segment (B, arrow). Magnetic resonance imaging showed decreased flowrelated enhancement in this region on 3D TOF (C, arrow), an LRNC on T2-weighted images (D, arrow), and plaque enhancement on DANTE T1 SPACE postcontrast black blood images (E, arrow).

Two-dimensional or 3D techniques can be used in VW imaging and can be performed on 1.5-T scanners; however, 3-T scanners efficiently provide the high resolution and small voxel sizes needed to optimize these techniques. ${ }^{9}$ Two-dimensional techniques use spin echo sequences to generate blood suppression (dark blood) but cannot create

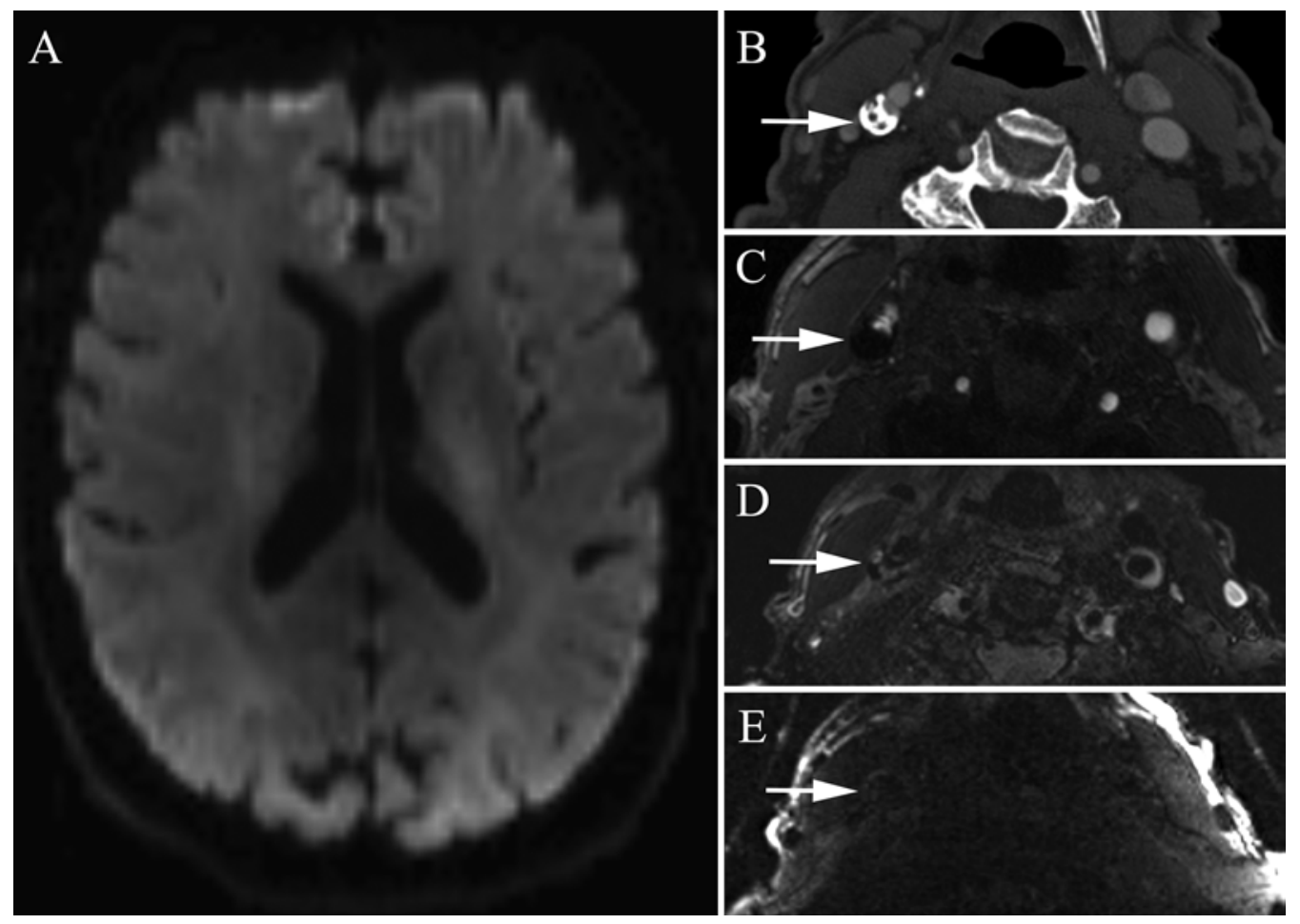

FIG. 4. A 70-year-old man with asymptomatic carotid stenosis on a screening examination performed prior to a lung transplant had hypertension and hyperlipidemia, for which he was on maximal medical therapy including dual antiplatelets (aspirin and clopidogrel), antihypertensive medications (hydrochlorothiazide and losartan), and a lipid-lowering agent (rosuvastatin). The patient had no evidence of infarct on diffusion-weighted images (A). Computed tomography angiography showed a large right carotid bifurcation plaque with $70 \%$ stenosis according to the North American Symptomatic Carotid Endarterectomy Trial criteria and a significant amount of calcification (B, arrow). Magnetic resonance imaging demonstrated a large right carotid plaque with low signal on TOF images (C, arrow), a T2-hyperintense LRNC and adjacent T2-hypointense calcification on T2-weighted images (D, arrow), and a lack of carotid IPH on MPRAGE images (E, arrow). 

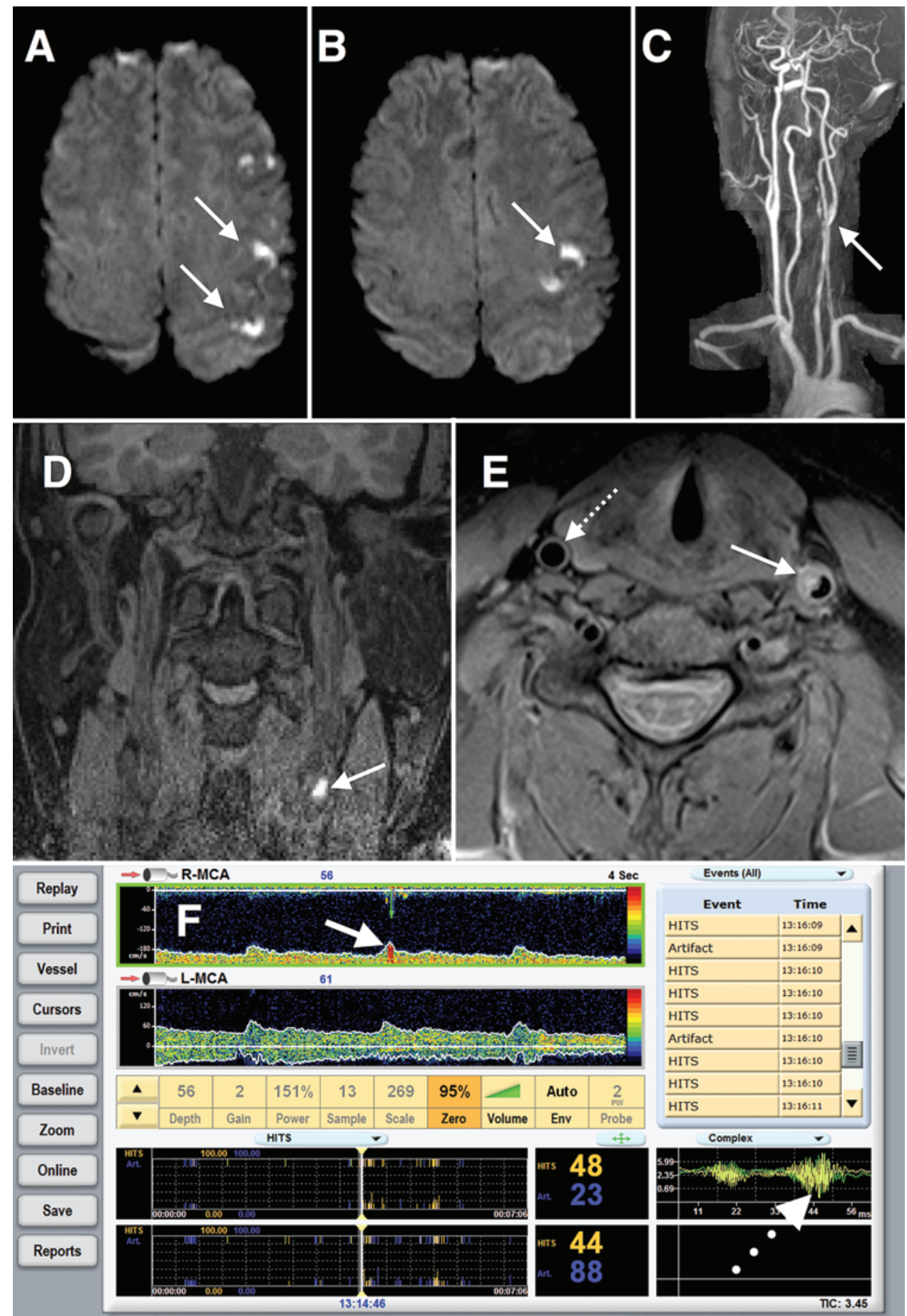

FIG. 5. Axial diffusion-weighted MRI revealing punctate infarcts (A and B, arrows) in the left MCA region. Contrast-enhanced MRA of the neck (C) showing mild stenosis of the left proximal ICA. Coronal MPRAGE image (D) showing an ICA hyperintensity (arrow) consistent with IPH. Proton density-weighted axial image $(\mathrm{E})$ confirming an intramural hyperintensity (arrow) of the left ICA, suggesting IPH and a vulnerable plaque, compared with the normal contralateral ICA (dotted arrow). Transcranial Doppler ultrasonography screenshot (F) showing an MES (arrow) and the automated Doppler analysis (dotted arrow).

blood suppression in slow or in-plane flow. ${ }^{9}$ To help provide a more accurate assessment of VW pathology, multiplanar 2D and 3D techniques are employed, and multiple sequences have been developed for this specific purpose. 9 Fortunately, the acquisition of sequences for VW imaging does not significantly increase the time required to perform scanning, with some sequences adding only 7-11 minutes.., 36

Vessel wall MRI began primarily with imaging of the extracranial carotid bifurcation plaque and was highly successful in detecting the features of unstable plaques that had been identified during histological analysis (for 
example, IPH). ${ }^{4}$ Both IPH and lipid-rich necrotic cores (LRNCs) are hyperintense on T1-weighted images, making it difficult to distinguish the two. ${ }^{12}$ Multicontrast techniques were initially used to help separate them; for instance, IPH is hyperintense on both T1-weighted and TOF images and thus can be separated from LRNC, which is hyperintense on T1-weighted images but isointense on TOF images. ${ }^{12}$ Fat-saturated sequences can also be used to saturate out the signal from the LRNC. ${ }^{4} \mathrm{Be}-$ cause of the difficulties in detecting IPH, researchers developed heavily T1-weighted sequences, such as the 3D magnetization-prepared rapid acquisition gradient echo (MPRAGE) sequence, which can detect IPH with high sensitivity, specificity, and accuracy (Figs. 1 and 3-5). ${ }^{34}$ The MPRAGE sequence selectively detects IPH as opposed to the LRNC, with the added benefit of having high inter- and intrarater reliability ${ }^{29}$ Because these 3D heavily T1-weighted sequences can be limited by motion and flow-related artifact, cine-MPRAGE and radially acquired stack of stars (SOS) MPRAGE were developed to allow easy separation of IPH from artifact. ${ }^{21,30}$

Plaque enhancement is also an important marker that can be used as a surrogate for an unstable plaque by representing neovascularization and macrophage infiltration. ${ }^{4}$ Evaluation of the fibrous cap and its correlate, plaque rupture, does not necessarily require high-resolution MRI and can often be performed with contrast-enhanced MRA; however, high-resolution sequences consisting of 3D multiple overlapping thin-slab angiography sequences have also been used., ${ }^{4}, 12$

Multiple studies have shown that the presence of IPH, LRNC, and/or thin fibrous caps, independently or in conjunction, may help to identify symptomatic plaques (Figs. 1-3). $2,4,28,31,35,42,44$ This was also confirmed in 3 recent meta-analyses, which all found an increased risk of future stroke (annual event risk 15\%-34\%) in patients with MPRAGE-positive IPH. ${ }^{15,17,39}$ Several studies have also shown that IPH is an independent risk factor for transient ischemic attack or stroke independent of a high degree of stenosis. ${ }^{2,4,28,42}$ These imaging characteristics have also been found in asymptomatic carotid artery lesions, and an LRNC and IPH, alone or together, have been shown to be associated with future ischemic events in asymptomatic patients as well (Fig. 4). ${ }^{31,42,44}$ In addition, patients with a higher percentage of LRNCs were found to have progressive stenosis of the involved vessel..$^{31}$ Many of the same characteristics of unstable carotid plaques (that is, plaque enhancement, enlargement, irregularity, and IPH) have been found to identify unstable intracranial plaques (Fig. 2). ${ }^{11,36}$

After the initial development of VW sequences to study the extracranial carotid artery, attention shifted to intracranial disease. Intracranial atherosclerotic plaque was a historically under-appreciated stroke source, but it is now known to have a high repeat-stroke risk despite maximal medical therapy.? In the past, it was often difficult to identify these lesions because they were frequently missed with lumen imaging techniques; however, with the advent of VW-MRI for intracranial vessels, lesion identification is becoming easier (Fig. 2).

Natori et al. ${ }^{33}$ used 3D T1-weighted fast-spin echo tech- niques for 3D VW imaging in 18 patients with acute middle cerebral artery (MCA) ischemic stroke. In 17 (94\%) of the 18 patients, this imaging technique demonstrated wall thickening suggestive of an MCA plaque, whereas MRA demonstrated $>50 \%$ stenosis in only 1 individual $(5.9 \%$, $\mathrm{p}<0.001$ ). Additionally, de Havenon et al. ${ }^{11}$ identified a symptomatic, nonstenotic intracranial atherosclerotic plaque on high-resolution MRI that was missed on conventional MRI, MRA, CTA, and DSA. High-resolution VW-MRI is more effective than luminal imaging at identifying symptomatic lesions in the posterior circulation and has the ability to detect symptomatic basilar artery atherosclerotic disease more frequently than MRA. ${ }^{23}$

Multiple studies using VW-MRI have shown that symptomatic intracranial lesions often have imaging characteristics similar to those of symptomatic extracranial lesions. ${ }^{11,36}$ In a study utilizing 3D, high-spatial-resolution, contrast-enhanced black-blood MRI, "culprit plaques" were thicker and demonstrated a greater degree and higher grade of contrast enhancement than "probable culprit plaques" and "non-culprit plaques." 11,36 Zhao et al. ${ }^{47}$ found that symptomatic plaques were also more likely to have an irregular surface $(\mathrm{p}=0.036)$ and to be larger than asymptomatic plaques $(\mathrm{p}=0.033)$. Additionally, symptomatic plaques, as diagnosed by increased plaque signal intensity, were more likely to have IPH (57.1\%) than the asymptomatic plaques $(22 \%)$. $^{38}$

Vasculitis, like intracranial atherosclerosis, is another stroke source that has been traditionally difficult to diagnosis using lumen imaging, with definitive diagnosis requiring invasive procedures such as lumbar puncture and/ or brain biopsy. ${ }^{9}$ However, VW-MRI is helping to make diagnosis easier by detecting the VW enhancement that is nearly universally present in this condition. This enhancement is a result of the mural inflammation that is a hallmark of this disease process. ${ }^{9,25}$ Vessel wall MRI is not only helpful in diagnosing vasculitis, but also useful in differentiating vasculitis from other intracranial vascular pathologies. ${ }^{32}$ The enhancement in vasculitis is typically concentric, although it is usually eccentric in atherosclerotic lesions or absent in cases of reversible cerebral vasospasm syndrome..$^{26,32}$ Vasculitis, unlike atherosclerotic lesions, rarely demonstrates T2 hyperintensity. ${ }^{32}$ Vessel wall MRI in vasculitis may also track the response to treatment over time. Current data have shown mixed results for this capability, however, with some studies showing resolution of enhancement over time and others demonstrating no significant change in the enhancement pattern. ${ }^{9}$

\section{Transcranial Doppler Ultrasonography}

A minimally invasive and inexpensive modality, TCD ultrasonography can be used in the diagnosis of many different causes of acute ischemic stroke. It is performed using a low-frequency transducer $(\leq 2 \mathrm{mHz})$ placed on the scalp at 4 sonographic windows that allow insonation of all major vessels in the circle of Willis. The transducer is often temporarily secured to the patient's head in a fixed position to allow for more consistent readings. ${ }^{8}$ The temporal window allows insonation of the MCA, anterior cerebral artery, proximal posterior cerebral arteries, and 
proximal internal carotid artery (ICA). The occipital window is primarily for insonation of the vertebral arteries and basilar artery. The submandibular window is used for the external ICA, and the orbital window for the ophthalmic artery.

Transcranial Doppler ultrasonography is commonly used during the workup for acute ischemic stroke to diagnose paradoxical emboli in the setting of a PFO. An ultrasonic contrast medium, typically agitated saline, is injected intravenously while the patient rests and performs a Valsalva maneuver to potentially reverse the normal flow through the PFO and create a right-to-left shunt. As the agitated saline is injected, the transducer is placed within the temporal sonographic window to insonate the MCA. ${ }^{8}$ The detection of any microembolic signals (MESs) is diagnostic of a PFO. The number of MESs has been proposed to represent the severity of the PFO shunting and, consequently, a higher risk of future ischemic stroke. ${ }^{8}$ Transcranial Doppler ultrasonography can also be used to detect the less common right-to-left shunts, such as in pulmonary arteriovenous fistulas. ${ }^{8}$

de Havenon et al. ${ }^{10}$ found that TCD ultrasonography performs comparably to more conventional methods for PFO detection. It had higher sensitivity than echocardiography, diagnosing an additional $15 \%-25 \%$ of patients with a PFO than would be seen with cardiac ultrasonography. This finding was also supported by a meta-analysis performed by Katsanos et al. ${ }^{20}$ who compared TCD ultrasound detection of a PFO with transthoracic echocardiography (TTE) detection. The authors found that TCD had a higher sensitivity ( $96.1 \%$ vs $45.1 \%$ ) than TTE but a lower specificity (92.4\% vs $99.6 \%$ ), negative likelihood ratio (0.04 vs 0.55 ), and positive likelihood ratio (12.62 vs 106.61). Given these findings, the authors suggested that the diagnostic yield of TCD ultrasonography was higher than that of TTE, thus providing a more cost-effective tool for PFO detection. ${ }^{20}$

The ability of TCD ultrasonography to detect MESs also allows the imaging technique to be used in the diagnosis of symptomatic carotid artery atherosclerotic lesions (Fig. 5). One study revealed that $43 \%$ of symptomatic carotid lesions demonstrated MESs, whereas only $10 \%$ of asymptomatic lesions did. ${ }^{37}$ In addition, in patients with symptomatic carotid plaques, MESs predicted stroke alone (OR 9.57, 95\% CI 1.54-59.38, $\mathrm{p}=0.02$ ) and combined with transient ischemic attack (OR 6.36, 95\% CI 2.90-13.96, p $<0.00001) .^{22}$ Patients with asymptomatic carotid lesions who are found to have MESs are also significantly more likely to experience a stroke within 1 year with an OR of 7.46 (95\% CI 2.24-24.89, $\mathrm{p}=0.001)$ for stroke alone and $12.00(95 \%$ CI $2.43-59.34, \mathrm{p}=0.002)$ for stroke/transient ischemic attack. ${ }^{22,43}$

In the Clopidogrel and Aspirin for Reduction of Emboli in Symptomatic Carotid Stenosis (CARESS) trial, investigators found that 7 days after the start of treatment only $43.8 \%$ of patients with symptomatic carotid stenosis who were started on dual antiplatelet therapy had MESs, whereas $72.7 \%$ of those started on aspirin alone had MESs ( $\mathrm{RR}$ reduction $39.8 \%, 95 \%$ CI $13.8-58.0, \mathrm{p}=0.0046) .{ }^{27}$ The MES frequency per hour, as compared with baseline, was also significantly reduced by $61.4 \%(95 \% \mathrm{CI}$ $31.6-78.2, p=0.0013)$ at 7 days after initiation of treatment with clopidogrel and aspirin and by $61.6 \%$ (95\% CI
34.9-77.4, $\mathrm{p}=0.0005$ ) at 2 days after initiation of treatment. Additionally, van Zuilen et al ${ }^{46}$ identified a significant reduction in MES after carotid endarterectomy. Thus, TCD ultrasonography, in conjunction with other imaging studies, can help to identify symptomatic carotid lesions and their future risk. ${ }^{4}$ Transcranial Doppler ultrasonography has also been used in the diagnosis of acute ischemic strokes in the setting of spontaneous cervical arterial dissections. Brunser et al. ${ }^{5}$ found that the presence of an MES and abnormal vasomotor reactivity, as determined by an abnormal breath hold index, was significantly predictive of a future ischemic event in these patients.

Transcranial Doppler ultrasonography is also a useful adjunct to traditional imaging techniques in the diagnosis and management of acute intracranial large vessel occlusion. This imaging technique correctly identified MCA occlusions with a sensitivity and specificity $\geq 90 \% .{ }^{8,45} \mathrm{Al}-$ though it was less sensitive than CTA (33\%-75\%) in identifying a thrombus within the carotid terminus and basilar artery, it was equally specific (99\%-100\%) in detecting occlusion in the MCA. ${ }^{45}$ Transcranial Doppler ultrasonography has primarily been used to detect arterial occlusions or collateral flow but can also be used to detect re-stenosis or re-occlusion after successful thrombectomy. ${ }^{6,8}$ It can augment CTA data by providing real-time assessments of flow velocity and ongoing emboli. ${ }^{45}$ However, the lack of an adequate sonographic window can limit the use of TCD ultrasonography in acute ischemic stroke, with approximately $10 \%$ of patients having a temporal bone thickness that precludes the use of ultrasonography. ${ }^{3,6,45}$ The utility of ultrasonography is also limited by the need for an experienced clinician or technician to perform and interpret the images. ${ }^{6,45}$

The usefulness of TCD ultrasonography in addition to noncontrast CT, CTA, and MRI diffusion in the workup for acute ischemic stroke was confirmed by Brunser et al. ${ }^{6}$ In $57 \%$ of cases, the imaging procedure provided additional useful information, including that on collateral flow, active microemboli, vessel patency, and detection of occlusion initially missed on CTA without CT perfusion. In $19.7 \%$ of cases, it provided more than one piece of additional information. Transcranial Doppler ultrasonography was also used to evaluate the efficacy of intravenous recombinant tissue plasminogen activator administration and any need for subsequent mechanical thrombectomy.

\section{Four-Dimensional CTA and MRA}

Conventional CTA and MRA are integral to the workup for acute ischemic stroke by helping to identify the location of large vessel occlusions. Because these imaging modalities lack temporal resolution and cannot provide dynamic information regarding blood flow or collateral flow to ischemic areas, their diagnostic capabilities have been primarily limited to thrombus detection. Four-dimensional CTA and MRA attempt to address this shortcoming by providing a dynamic component to conventional CTA and MRA, thus allowing for temporal resolution.

The dynamic aspect is accomplished by taking multiple sequential images of the same anatomical territory. ${ }^{24}$ In $4 \mathrm{D}$ CTA, images are taken throughout the administration of a contrast bolus, whereas 4D MRA can be performed with or without contrast administration. ${ }^{16,18,19,24,40,41}$ Although 
the initial experience with 4D MRA involved contrast administration, noncontrast-enhanced sequences such as 4D arterial spin-labeling MRA and 4D TOF have been developed, thus avoiding any potential complications from the use of contrast..$^{19,40,41}$ Multiple studies have shown that both contrast-enhanced and non-contrast-enhanced 4D MRA can quantify the degree of collateral circulation to an extent comparable with DSA, which is considered the gold standard. ${ }^{16,18}$

Improved collateral flow surrounding an area of ischemia has been associated with better rates of recanalization, a lower incidence of hemorrhagic transformation, and improved clinical outcome after both thrombectomy and thrombolytic administration. ${ }^{18,24}$ Therefore, the ability of 4D CTA and MRA to accurately determine the extent of collateral blood flow can help to guide the management of acute ischemic stroke. In addition, 4D imaging has been shown to better detect the extent of a thromboembolus and any trace anterograde flow past a thromboembolus, both of which are associated with an increased rate of recanalization after thrombolytic administration. ${ }^{13,14}$ Despite its many advantages, 4D imaging is limited by its prolonged image acquisition times and, in the setting of CT, a higher radiation dose than in routine CTA. ${ }^{24,41}$

\section{Conclusions}

As technological advances in the imaging sciences occur, the role of new imaging techniques in the evaluation and management of acute ischemic stroke evolves. Vessel wall MRI, TCD ultrasonography, and 4D CTA or MRA are 3 of the newer imaging modalities in use today that have proven utility in this patient cohort. Although each of the modalities has a set of limitations, it is likely that their roles will continue to expand in the future.

\section{References}

1. Adams HP Jr, Bendixen BH, Kappelle LJ, Biller J, Love $\mathrm{BB}$, Gordon DL, et al: Classification of subtype of acute ischemic stroke. Definitions for use in a multicenter clinical trial. TOAST. Trial of Org 10172 in Acute Stroke Treatment. Stroke 24:35-41, 1993

2. Altaf N, Daniels L, Morgan PS, Auer D, MacSweeney ST, Moody AR, et al: Detection of intraplaque hemorrhage by magnetic resonance imaging in symptomatic patients with mild to moderate carotid stenosis predicts recurrent neurological events. J Vasc Surg 47:337-342, 2008

3. Best LM, Webb AC, Gurusamy KS, Cheng SF, Richards T: Transcranial Doppler ultrasound detection of microemboli as a predictor of cerebral events in patients with symptomatic and asymptomatic carotid disease: a systematic review and meta-analysis. Eur J Vasc Endovasc Surg 52:565-580, 2016

4. Brinjikji W, Huston J III, Rabinstein AA, Kim GM, Lerman A, Lanzino G: Contemporary carotid imaging: from degree of stenosis to plaque vulnerability. J Neurosurg 124:27-42, 2016

5. Brunser AM, Lavados PM, Hoppe A, Muñoz-Venturelli P, Sujima E, López J, et al: Transcranial Doppler as a predictor of ischemic events in carotid artery dissection. J Neuroimaging [epub ahead of print], 2016

6. Brunser AM, Mansilla E, Hoppe A, Olavarría V, Sujima E, Lavados PM: The role of TCD in the evaluation of acute stroke. J Neuroimaging 26:420-425, 2016

7. Chimowitz MI, Lynn MJ, Derdeyn CP, Turan TN, Fiorella D,
Lane BF, et al: Stenting versus aggressive medical therapy for intracranial arterial stenosis. N Engl J Med 365:993-1003, 2011

8. D'Andrea A, Conte M, Cavallaro M, Scarafile R, Riegler L, Cocchia R, et al: Transcranial Doppler ultrasonography: From methodology to major clinical applications. World J Cardiol 8:383-400, 2016

9. de Havenon A, Chung L, Park M, Mossa-Basha M: Intracranial vessel wall MRI: a review of current indications and future applications. Neurovascular Imaging 2:10, 2016

10. de Havenon A, Moore A, Sultan-Qurraie A, Majersik JJ, Stoddard G, Tirschwell D: Ischemic stroke patients with active malignancy or extracardiac shunts are more likely to have a right-to-left shunt found by TCD than echocardiogram. Transl Stroke Res 6:361-364, 2015

11. de Havenon A, Yuan C, Tirschwell D, Hatsukami T, Anzai Y, Becker K, et al: Nonstenotic culprit plaque: the utility of high-resolution vessel wall MRI of intracranial vessels after ischemic stroke. Case Rep Radiol 2015:356582, 2015

12. den Hartog AG, Bovens SM, Koning W, Hendrikse J, Luijten PR, Moll FL, et al: Current status of clinical magnetic resonance imaging for plaque characterisation in patients with carotid artery stenosis. Eur J Vasc Endovasc Surg 45:7-21, 2013

13. Frölich AM, Psychogios MN, Klotz E, Schramm R, Knauth M, Schramm P: Antegrade flow across incomplete vessel occlusions can be distinguished from retrograde collateral flow using 4-dimensional computed tomographic angiography. Stroke 43:2974-2979, 2012

14. Frölich AM, Schrader D, Klotz E, Schramm R, Wasser K, Knauth M, et al: 4D CT angiography more closely defines intracranial thrombus burden than single-phase CT angiography. AJNR Am J Neuroradiol 34:1908-1913, 2013

15. Gupta A, Baradaran H, Schweitzer AD, Kamel H, Pandya A, Delgado D, et al: Carotid plaque MRI and stroke risk: a systematic review and meta-analysis. Stroke 44:3071-3077, 2013

16. Hernández-Pérez M, Puig J, Blasco G, Pérez de la Ossa N, Dorado L, Dávalos A, et al: Dynamic magnetic resonance angiography provides collateral circulation and hemodynamic information in acute ischemic stroke. Stroke 47:531-534, 2016

17. Hosseini AA, Kandiyil N, MacSweeney STS, Altaf N, Auer DP: Carotid plaque hemorrhage on magnetic resonance imaging strongly predicts recurrent ischemia and stroke. Ann Neurol 73:774-784, 2013

18. Iryo Y, Hirai T, Nakamura M, Inoue Y, Watanabe M, Ando Y, et al: Collateral circulation via the circle of Willis in patients with carotid artery steno-occlusive disease: evaluation on 3-T 4D MRA using arterial spin labelling. Clin Radiol 70:960-965, 2015

19. Iryo Y, Hirai T, Nakamura M, Tateishi M, Hayashida E, Azuma M, et al: Evaluation of intracranial vasculatures in healthy subjects with arterial-spin-labeling-based 4D-MR angiography at 3T. Magn Reson Med Sci 15:335-339, 2016

20. Katsanos AH, Psaltopoulou T, Sergentanis TN, Frogoudaki A, Vrettou AR, Ikonomidis I, et al: Transcranial Doppler versus transthoracic echocardiography for the detection of patent foramen ovale in patients with cryptogenic cerebral ischemia: A systematic review and diagnostic test accuracy meta-analysis. Ann Neurol 79:625-635, 2016

21. Kim SE, Roberts JA, Eisenmenger LB, Aldred BW, Jamil $\mathrm{O}$, Bolster BD, et al: Motion-insensitive carotid intraplaque hemorrhage imaging using 3D inversion recovery preparation stack of stars (IR-prep SOS) technique. J Magn Reson Imaging 45:410-417, 2017

22. King A, Markus HS: Doppler embolic signals in cerebrovascular disease and prediction of stroke risk: a systematic review and meta-analysis. Stroke 40:3711-3717, 2009 
23. Klein IF, Lavallée PC, Mazighi M, Schouman-Claeys E, Labreuche J, Amarenco P: Basilar artery atherosclerotic plaques in paramedian and lacunar pontine infarctions: a high-resolution MRI study. Stroke 41:1405-1409, 2010

24. Kortman HG, Smit EJ, Oei MT, Manniesing R, Prokop M, Meijer FJ: 4D-CTA in neurovascular disease: a review. AJNR Am J Neuroradiol 36:1026-1033, 2015

25. Küker W, Gaertner S, Nagele T, Dopfer C, Schoning M, Fiehler J, et al: Vessel wall contrast enhancement: a diagnostic sign of cerebral vasculitis. Cerebrovasc Dis 26:23-29, 2008

26. Mandell DM, Matouk CC, Farb RI, Krings T, Agid R, terBrugge K, et al: Vessel wall MRI to differentiate between reversible cerebral vasoconstriction syndrome and central nervous system vasculitis: preliminary results. Stroke 43:860-862, 2012

27. Markus HS, Droste DW, Kaps M, Larrue V, Lees KR, Siebler $\mathrm{M}$, et al: Dual antiplatelet therapy with clopidogrel and aspirin in symptomatic carotid stenosis evaluated using Doppler embolic signal detection: the Clopidogrel and Aspirin for Reduction of Emboli in Symptomatic Carotid Stenosis (CARESS) trial. Circulation 111:2233-2240, 2005

28. McNally JS, McLaughlin MS, Hinckley PJ, Treiman SM, Stoddard GJ, Parker DL, et al: Intraluminal thrombus, intraplaque hemorrhage, plaque thickness, and current smoking optimally predict carotid stroke. Stroke 46:84-90, 2015

29. McNally JS, Yoon HC, Kim SE, Narra KK, McLaughlin MS, Parker DL, et al: Carotid MRI Detection of Intraplaque Hemorrhage at 3T and 1.5T. J Neuroimaging 25:390-396, 2015

30. Mendes J, Parker DL, Kim SE, Treiman GS: Reduced blood flow artifact in intraplaque hemorrhage imaging using CineMPRAGE. Magn Reson Med 69:1276-1284, 2013

31. Mono ML, Karameshev A, Slotboom J, Remonda L, Galimanis A, Jung S, et al: Plaque characteristics of asymptomatic carotid stenosis and risk of stroke. Cerebrovasc Dis 34:343350,2012

32. Mossa-Basha M, Hwang WD, De Havenon A, Hippe D, Balu N, Becker KJ, et al: Multicontrast high-resolution vessel wall magnetic resonance imaging and its value in differentiating intracranial vasculopathic processes. Stroke 46:1567-1573, 2015

33. Natori T, Sasaki M, Miyoshi M, Ohba H, Katsura N, Yamaguchi M, et al: Evaluating middle cerebral artery atherosclerotic lesions in acute ischemic stroke using magnetic resonance T1-weighted 3-dimensional vessel wall imaging. J Stroke Cerebrovasc Dis 23:706-711, 2014

34. Ota H, Yarnykh VL, Ferguson MS, Underhill HR, Demarco JK, Zhu DC, et al: Carotid intraplaque hemorrhage imaging at 3.0-T MR imaging: comparison of the diagnostic performance of three T1-weighted sequences. Radiology 254:551563,2010

35. Qiao Y, Etesami M, Astor BC, Zeiler SR, Trout HH III, Wasserman BA: Carotid plaque neovascularization and hemorrhage detected by MR imaging are associated with recent cerebrovascular ischemic events. AJNR Am J Neuroradiol 33:755-760, 2012

36. Qiao Y, Zeiler SR, Mirbagheri S, Leigh R, Urrutia V, Wityk $\mathrm{R}$, et al: Intracranial plaque enhancement in patients with cerebrovascular events on high-spatial-resolution MR images. Radiology 271:534-542, 2014

37. Ritter MA, Dittrich R, Thoenissen N, Ringelstein EB, Nabavi DG: Prevalence and prognostic impact of microembolic sig- nals in arterial sources of embolism. A systematic review of the literature. J Neurol 255:953-961, 2008

38. Ryu CW, Jahng GH, Kim EJ, Choi WS, Yang DM: High resolution wall and lumen MRI of the middle cerebral arteries at 3 tesla. Cerebrovasc Dis 27:433-442, 2009

39. Saam T, Hetterich H, Hoffmann V, Yuan C, Dichgans M, Poppert H, et al: Meta-analysis and systematic review of the predictive value of carotid plaque hemorrhage on cerebrovascular events by magnetic resonance imaging. J Am Coll Cardiol 62:1081-1091, 2013

40. Sakai M, Illies T, Jerusel N, Tateishi S, Uchikoshi M, Fiehler $\mathrm{J}$, et al: Feasibility of non-contrast-enhanced four dimensional (4D) MRA in head and neck tumors, comparison with contrast-enhanced 4D MRA. Springerplus 5:1282, 2016

41. Shibukawa S, Nishio H, Niwa T, Obara M, Miyati T, Hara $\mathrm{T}$, et al: Optimized 4D time-of-flight MR angiography using saturation pulse. J Magn Reson Imaging 43:1320-1326, 2016

42. Singh N, Moody AR, Gladstone DJ, Leung G, Ravikumar R, Zhan J, et al: Moderate carotid artery stenosis: MR imagingdepicted intraplaque hemorrhage predicts risk of cerebrovascular ischemic events in asymptomatic men. Radiology 252:502-508, 2009

43. Spence JD, Tamayo A, Lownie SP, Ng WP, Ferguson GG: Absence of microemboli on transcranial Doppler identifies low-risk patients with asymptomatic carotid stenosis. Stroke 36:2373-2378, 2005

44. Takaya N, Yuan C, Chu B, Saam T, Underhill H, Cai J, et al: Association between carotid plaque characteristics and subsequent ischemic cerebrovascular events: a prospective assessment with MRI--initial results. Stroke 37:818-823, 2006

45. Tsivgoulis G, Sharma VK, Lao AY, Malkoff MD, Alexandrov AV: Validation of transcranial Doppler with computed tomography angiography in acute cerebral ischemia. Stroke 38:1245-1249, 2007

46. van Zuilen EV, Moll FL, Vermeulen FE, Mauser HW, van Gijn J, Ackerstaff RG: Detection of cerebral microemboli by means of transcranial Doppler monitoring before and after carotid endarterectomy. Stroke 26:210-213, 1995

47. Zhao DL, Deng G, Xie B, Ju S, Yang M, Chen XH, et al: High-resolution MRI of the vessel wall in patients with symptomatic atherosclerotic stenosis of the middle cerebral artery. J Clin Neurosci 22:700-704, 2015

\section{Disclosures}

Dr. Taussky has served as a consultant to Covidien/Medtronic.

\section{Author Contributions}

Conception and design: Park, McNally, de Havenon. Drafting the article: Kilburg, McNally, de Havenon. Critically revising the article: Park, McNally, de Havenon, Taussky, Kalani. Reviewed submitted version of manuscript: all authors.

\section{Correspondence}

Min S. Park, Department of Neurosurgery, Clinical Neurosciences Center, University of Utah, 175 N Medical Dr. East, Salt Lake City, UT 84132. email: neuropub@hsc.utah.edu. 